Case report

\title{
Pulmonary vein thrombosis secondary to tuberculosis in a non-HIV infected patient
}

\author{
Yonas Raru ${ }^{\mathrm{a}, *}$, Jason Ballengee ${ }^{\mathrm{a}}$, Mahmoud Abouzid ${ }^{\mathrm{a}}$, Fuad Zeid ${ }^{\mathrm{b}}$, Samson Teka \\ ${ }^{a}$ Internal Medicine Resident, Marshall University School of Medicine, Huntington, WV, USA \\ ${ }^{\mathrm{b}}$ Pulmonary and Critical Care Medicine, Marshall University School of Medicine, Huntington, WV, USA \\ ${ }^{\mathrm{c}}$ Department of Internal Medicine, Marshall University School of Medicine and Huntington VAMC, Huntington, WV, USA
}

\section{A B S T R A C T}

Tuberculosis has been suggested as an independent risk factor for thromboembolism due to a hypercoagulable state induced by changes in clotting factors, protein C and vascular endothelium. Pulmonary vein thrombosis (PVT) is a rare, potentially serious and life-threatening condition that can be caused by tuberculosis. Its rare occurrence is due to a rich network of venous collateral vessels that drain the lung. PVT can also occur following lobectomy for malignancy, lung transplantation, radiofrequency catheter ablation for atrial fibrillation, sclerosing mediastinitis and following metastatic cancer, such as liposarcoma. Pulmonary vein thrombosis is difficult to diagnose clinically and requires a combination of conventional diagnostic modalities. Systemic anticoagulation, treatment of the predisposing pathology and monitoring of the thrombosis are parts of management of pulmonary vein thrombosis. We present a case of pulmonary vein thrombosis due to tuberculosis. Our patient is a 67-year-old Caucasian male with a past medical history significant for hypertension and hyperlipidemia who came to our hospital with hemoptysis, cough and shortness of breath 1-month duration. He also noticed around 20 pounds of weight loss and night sweats but had no fever. He had no history of travel outside the USA and has never been incarcerated before, but he endorsed that his son has been in Jail before but the son has never been diagnosed with TB. He quit smoking 2 months ago. He was tachycardic, tachypneic and had decreased air entry with crepitation in the right side of the posterior chest. Chest $\mathrm{x}$ ray showed multiple cavitary lesions in the right upper lobe area. CT scan of the chest with contrast showed pulmonary venous partial thrombosis in the right upper lobe with multiple cavitary lesions with hilar and mediastinal lymphadenopathy. Sputum AFB was positive for mycobacterium tuberculosis and was sensitive for rifampicin. Patient was treated with systemic anticoagulation and anti-tuberculosis medications. Patient's hospital course was complicated by development of elevated liver enzymes at which time the anti-TB medications were stopped to be restarted one by one with a follow up of his liver enzymes and liver function tests. Our case shows that presentation of patients with PVT is non-specific and needs a very high index of suspicion for proper diagnosis and management to prevent further complications as it is associated with limb and life-threatening complications. It also illustrates the importance of considering PVT in a patient with tuberculosis.

\section{Introduction}

About 30 percent of the world population are estimated to be infected with mycobacterium tuberculosis [1-3]. Tuberculosis has been suggested as an independent risk factor for thromboembolism due to a hypercoagulable state induced by changes in clotting factors, protein $\mathrm{C}$ and vascular endothelium [4,5]. Pulmonary vein thrombosis (PVT) is a rare, potentially serious and life-threatening condition that can be caused by tuberculosis. Its rare occurrence is due to a rich network of venous collateral vessels that drain the lung [6-8]. PVT can also occur following lobectomy for malignancy, lung transplantation, radiofrequency catheter ablation for atrial fibrillation, sclerosing mediastinitis and following metastatic cancer, such as liposarcoma [9-11]. Pulmonary vein thrombosis is difficult to diagnose clinically and requires a combination of conventional diagnostic modalities. Systemic anticoagulation, treatment of the predisposing pathology and monitoring of the thrombosis are parts of management of pulmonary vein thrombosis [8]. We present a case of pulmonary vein thrombosis due to tuberculosis.

\section{Case presentation}

Our patient is a 67-year-old male with a past medical history significant for hypertension and hyperlipidemia who came to our hospital with hemoptysis. He was also having cough and shortness of breath for the last 1 month. He said that his hemoptysis was about 1 cup per day mixed with yellowish sputum. He noticed around 20 pounds of weight loss in the last 1 month. He also complained of night sweats but had no fever. He had no history of travel outside the USA. He has never been incarcerated before, but he endorsed that his son has been in Jail before and he visited him twice a year in patient's home. But he also said that his son has never been diagnosed with TB. He smoked 1.5 packs per day for the last 50 years and quit smoking 2 months ago. His medication includes hydrochlorothiazide, lisinopril, gabapentin, aspirin and

\footnotetext{
* Corresponding author.

E-mail address: raru@marshall.edu (Y. Raru).
} 


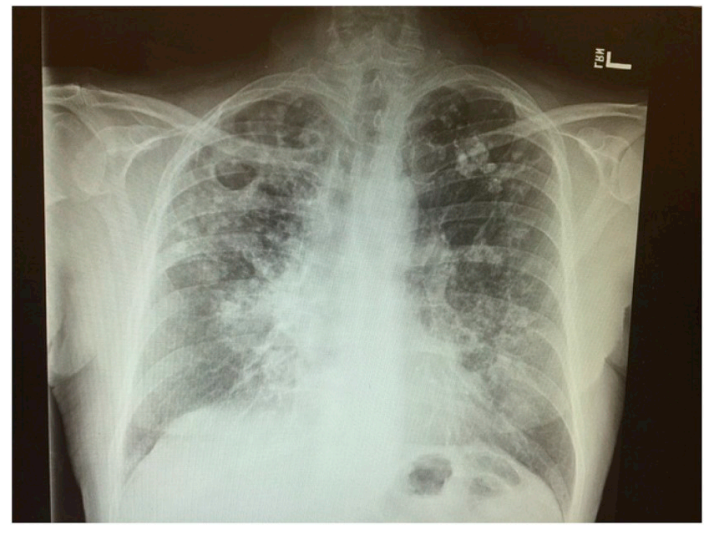

Fig. 1. Chest X ray.

trazodone. On examination, vital signs were within the normal range except a heart rate of 106 beats/minute. He had slightly pale conjunctiva, non-icteric sclera and had wet tongue and buccal mucosa. There was decreased air entry with crepitations in the right side of the posterior chest but no wheezes or rales. No peripheral lymphadenopathy, no peripheral edema or sign of fluid collection in the abdomen.

Chest $\mathrm{x}$ ray (Fig. 1) showed multiple cavitary lesion in the right upper lobe area. CT scan of the chest with contrast (Figs. 2 and 3) showed pulmonary venous partial thrombosis in the right upper lobe. Multiple cavitary lesions with hilar and mediastinal lymphadenopathy. There are also smaller nodular lesions in the left chest too. Small right pleural effusion with multiple calcified granulomata in the left upper lobe. QuantiFERON gold test was found to be positive. Sputum AFB smear was found to be strongly positive and it is sensitive to rifampin. Echocardiography showed no valvular lesions with preserved ejection fraction (> 65\%) and normal right ventricular size and normal right ventricular systolic pressure. Liver enzymes and renal function tests were found within the normal limit. HIV test was negative. Patient was started with intensive phase anti-tuberculosis treatment with rifampin, isoniazid, ethambutol, pyrazinamide with vitamin B6. He was also started with anticoagulation with heparin and warfarin considering the tuberculosis being the cause of the pulmonary vein thrombosis. Patient was also given supportive treatment and he made a gradual improvement and was discharged with anti-tuberculosis treatment and warfarin. Patient needed to be placed on a higher dose of warfarin as it was difficult to keep him therapeutic with lower doses. He was also advised to follow with infectious disease and anticoagulation clinic. Patient was found to have a significant increase in liver enzymes and bilirubin on follow up and the anti-TB medications were stopped to be restarted one by one with a follow up of his liver enzymes and liver function tests. He was also continued with warfarin.

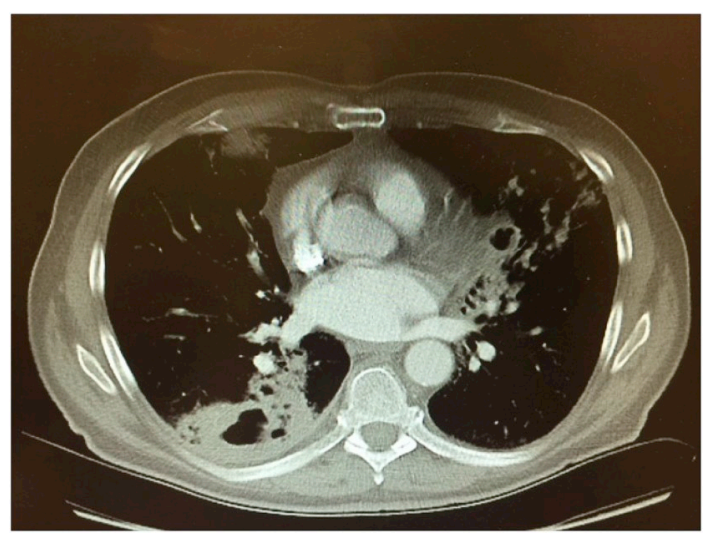

Fig. 2. CT scan showing the pulmonary vein thrombosis.

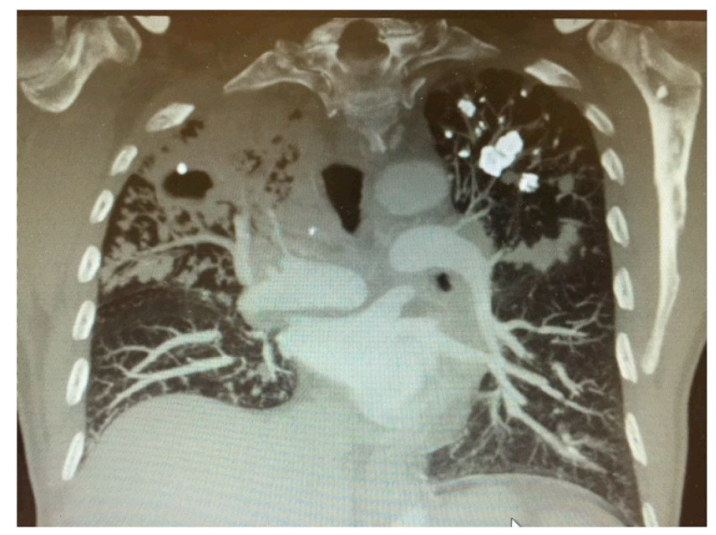

Fig. 3. CT scan picture showing the TB lesions and pulmonary vein thrombosis.

\section{Discussion}

More than two billion people, about 30 percent of the world population, are estimated to be infected with mycobacterium tuberculosis. The global incidence of tuberculosis (TB) peaked around 2003 and appears to be declining slowly. According to the World Health Organization (WHO), in 2016, 10.4 million individuals became ill with TB and 1.7 million died [1]. Poverty, human immunodeficiency virus (HIV), and drug resistance are major contributors to the resurging global TB epidemic. Approximately 95 percent of TB cases occur in developing countries (2). Around 70\% of TB cases in the United States now occurs in foreign-born individuals emigrating from countries with high rates of endemic TB [3]. Tuberculosis, both pulmonary and extrapulmonary, has been suggested as an independent risk factor for venous thromboembolism (VTE), perhaps due to a hypercoagulable state.

In one retrospective study including 3485 cases of active TB, the prevalence of VTE was approximately 2 percent, which is similar to the rate associated with malignancy and approximately 100 times higher than the incidence of VTE among hospitalized patients in general $[4,5]$. Previous studies have shown that there is high level of plasma fibrinogen with impaired fibrinolysis, low level of antithrombin III and protein $\mathrm{C}$ which induce a hypercoagulable state and thus more thrombosis. It has also been shown that there is significant decrease of prothrombin levels in one third of tuberculosis patients which also further exposes TB patients to thrombosis $[8,9]$. The other theory suggested in literature as to the cause of thrombosis in TB is the cytokine theory where cytokines are proinflammatory and activate the vascular intima and make the endothelium more thrombogenic [9]. Some authors have postulated that various local factors may lead to thrombosis such as from venous compression by lymph nodes, as mediastinal and retroperitoneal lymphadenopathies may cause chest or inferior vena cava thrombosis in the absence of any hemostatic abnormalities. However, there are also literatures suggesting that systemic rather than local factors play a predominant role [6]. There are case reports on different types of thrombosis in literature related to tuberculosis including extremity deep venous thrombosis, portal vein thrombosis, hepatic vein thrombosis and superior sagittal vein thrombosis [10-12]. Our patient presented with pulmonary vein thrombosis with a newly diagnosed tuberculosis. Pulmonary vein thrombosis is a rare condition. The incidence is unclear, as most of the literature includes case reports. The majority of cases are reported following lobectomy for malignancy, lung transplantation, radiofrequency catheter ablation for atrial fibrillation, sclerosing mediastinitis and following metastatic cancer, such as liposarcoma. Idiopathic cases of pulmonary vein thrombosis are also described in a literature. Most patients with PVT are commonly asymptomatic or have nonspecific symptoms such as cough, hemoptysis, and dyspnea from pulmonary edema or 
infarction. In majority of cases, pulmonary artery pressure increases concomitant with systemic hypotension and low cardiac output. Respiratory parameters also deteriorate, including worsening oxygenation, hypercapnia, and decreasing pulmonary compliance. Our patient presented with mild hemoptysis and shortness of breath but didn't have any hemodynamic abnormality other than a tachycardia of 106 beats per minute [8]. Our patient also didn't have an increase in pulmonary artery pressure. This can be explained by the partial nature of pulmonary vein obstruction seen in our patient. He was also having cough with yellowish sputum.

The coexistence of tuberculosis and pulmonary vein thrombosis in our patient will make it very difficult to say that a symptom is caused by TB or PVT as it could also be the other one causing the same symptom. One case report in literature described a presentation of tuberculosis with pulmonary vein thrombosis with atrial extension which we didn't see in our patient [13]. Post-operative, Malignancy and radiofrequency catheter ablation related pulmonary vein thromboses have a dramatic presentation with hemodynamic instability and it requires prompt diagnosis and intervention due to high mortality. The relatively stable presentation of our patient is due to the incomplete obstruction and the fact that the patient might have presented early, and this type of nonspecific presentation needs a high index of suspicion for the condition and proper investigation and management to prevent further complications. Pulmonary vein thrombosis is difficult to diagnose clinically and requires a combination of conventional diagnostic modalities. Pulmonary venous phase of a contrast CT of the chest is used to diagnose pulmonary vein thrombosis and our patient was diagnosed while undergoing a contrast CT scan. In literature, a trans esophageal echocardiogram (TEE) can also be used to diagnose it and TEE may demonstrate extension of a thrombus into the atrium if it is present. In malignancy associated pulmonary vein thrombosis, Magnetic resonance imaging of the chest is a useful modality for diagnosis, because it is able to distinguish between a bland thrombus and a tumor thrombus in the pulmonary vein [6-8]. Treatment of PVT depends on the overall clinical condition and comorbidity of the patient. There are no consensus guidelines on optimal management of PVT, but anticoagulation is very important even if there is no preferred duration and type of anticoagulant. It was also noticed in literatures that treating the underlying pathology whether it was post-operative, tuberculosis, or malignancy may lead to favorable outcomes in the pulmonary vein thrombosis. In some cases of postoperative patients in whom anticoagulant was contraindicated, spontaneous lysis of small, untreated thrombi without sequelae was observed. Thrombectomy has been successfully performed when medical therapy fails. Lobectomy may be indicated when PVT is complicated with massive hemoptysis or pulmonary necrosis $[6,8,14]$. Our patient was started with treatment with anti-tuberculosis medications and systemic anticoagulation. He responded to the treatment very well but developed elevated liver enzymes secondary to the anti-TB medications. The medications were discontinued to be restarted one by one when the liver enzymes improve. Our patient was started with heparin and warfarin and once he was therapeutic (INR $=2-3$ ), he was continued with warfarin.

He needed a higher daily dose of warfarin (10mg) because of the effect of rifampin on warfarin metabolism. Complications that can occur after PVT include pulmonary infarction, pulmonary edema and right ventricular failure. Peripheral embolism was also seen in literature including limb ischemia, stroke, and renal infarction. Because of these life and limb threatening complications, waiting for spontaneous lysis is very risky and it is suggested to put patients on systemic anticoagulation and monitoring of the thrombus size in the coming months [8].

\section{Conclusion}

Tuberculosis, both pulmonary and extrapulmonary, has been suggested as an independent risk factor for thromboembolism due to a hypercoagulable state induced by changes in clotting factors, protein $\mathrm{C}$ and vascular endothelium. Pulmonary vein thrombosis can be seen in patients with tuberculosis, lung transplantation, malignancy and post radiofrequency catheter ablation. Presentation of patients is non-specific and needs a very high index of suspicion for proper diagnosis and management to prevent further complications. Contrast enhanced CT and trans esophageal echocardiogram are important for confirming the diagnosis and evaluate atrial extension. Pulmonary vein thrombosis is associated with limb and life-threatening complications and needs early detection and treatment to prevent these complications. Systemic anticoagulation, treatment of the predisposing pathology and monitoring of the thrombosis are parts of management of pulmonary vein thrombosis.

\section{Conflict of interest}

The authors have no conflict of interest.

\section{References}

[1] World Health Organization, Global Tuberculosis Report, (2017).

[2] E.L. Corbett, B. Marston, G.J. Churchyard, K.M. De Cock, Tuberculosis in subSaharan Africa: opportunities, challenges, and change in the era of antiretroviral treatment, Lancet 367 (2006) 926.

[3] R.J. Stewart, C.A. Tsang, R.H. Pratt, et al., Tuberculosis - United States, 2017, MMWR Morb. Mortal. Wkly. Rep. 67 (2018) 317.

[4] C. Dentan, O. Epaulard, D. Seynaeve, et al., Active tuberculosis and venous thromboembolism: association according to international classification of diseases, ninth revision hospital discharge diagnosis codes, Clin. Infect. Dis. 58 (2014) 495.

[5] J.A. Heit, L.J. Melton 3rd, C.M. Lohse, et al., Incidence of venous thromboembolism in hospitalized patients vs community residents, Mayo Clin. Proc. 76 (2001) 1102.

[6] S. Bansal, K. Utpat, J.M. Joshi, Systemic thrombosis due to pulmonary tuberculosis, Natl. Med. J. India 30 (2017) 201-202.

[7] O. Akiode, G. Prakash, Pulmonary vein thrombosis associated with metastatic carcinoma, Fed. Pract. 31 (2014) 26-28.

[8] G. Chaaya, P. Vishnubhotla, Pulmonary vein thrombosis: a recent systematic review, in: A. Muacevic, J.R. Adler (Eds.), Cureus, vol. 9, 2017, p. e993, , https://doi. org/10.7759/cureus.993 (1).

[9] A. Sharma, V. Sharma, Abdominal aortic thrombosis and tuberculosis: an uncommon association, Gastroenterol. Rep. (Oxf.) 2 (2014) 311-312.

[10] M. Ambrosetti, M. Ferrarese, L.R. Codecasa, G. Besozzi, A. Sarassi, P. Viggiani, et al., AIPO/SMIRA TB Study Group. Incidence of venous thromboembolism in tuberculosis patients, Respiration 73 (2006) 396.

[11] J.A. Fiorot Júnior, A.C. Felício, M.M. Fukujima, C.A. Rodrigues, V.M. Morelli, D.M. Lourenço, et al., Tuberculosis: an uncommon cause of cerebral venous thrombosis? Arq. Neuropsiquiatr. 63 (3B) (2005) 852-854.

[12] D.G. Fullerton, A. Shrivastava, M. Munavvar, S. Jain, J. Howells, P. Macdowall, Pulmonary tuberculosis presenting with central retinal vein occlusion, Br. J. Ophthalmol. 91 (2007) 1714-1715.

[13] J.P. Wu, Q. Wu, Y. Yang, Z.Z. Du, H.F. Sun, Idiopathic pulmonary vein thrombosis extending to left atrium: a case report with a literature review, Chin. Med. J. (Engl.) 125 (2012) 1197-1200.

[14] G.R. Alexander, A. Reddi, D. Reddy, Idiopathic pulmonary vein thrombosis: a rare cause of massive hemoptysis, Ann. Thorac. Surg. 88 (2009) 281-283, https://doi. org/10.1016/j.athoracsur.2008.09.061. 